\title{
„Die Dinge singen höre ich gern.“ \\ Eine Untersuchung zum Aspekt der „Ursprünglich- keit“ in Martin Heideggers Dichtungs-Theorie
}

\author{
Lajos Mitnyán
}

„Die Dinge singen höre ich gern."

\begin{abstract}
An analysis of the aspect of „originality” in Martin Heidegger's theory of poetry Abstract

The question of the following analysis encompasses a subject that primarily refers to Martin Heidegger's poetry theorem, but at the same time also addresses a general problem of scientific theory, and as such transcends the limits of a Heidegger study. In addition to analysing the questions given by the philosopher, the consequences that arise from the thesis are considered, according to which a specific transition can be postulated between thinking as a discursive cognitive process and poetry as an aesthetic creative process. On the other hand, the present study aims to take account of the controversy that has arisen around Heidegger by assigning his late writings to a grey area of the science and attempting to deny them scientific discourse today.
\end{abstract}

Keywords: German literature and philosophy; literary theory; aesthetics; cultural studies; Martin Heidegger; Rainer Maria Rilke; poem analysis

Schlüsselwörter: Deutsche Literatur und Philosophie; Literaturtheorie; Ästhetik; Kulturwissenschaft; Martin Heidegger; Rainer Maria Rilke; Gedichtsanalyse

Subject-Affiliation in New CEEOL: Philosophy - Aesthetics

DOI: 10.36007/eruedu.2020.3.095-106

\section{Einführung}

Die Fragestellung der nachfolgenden Analyse umfasst einen Themenbereich, der sich zwar primär auf Martin Heideggers Dichtungs-Theorem bezieht, aber zu gleicher Zeit auch ein allgemein wissenschaftstheoretisches Problem anspricht, und als solche sich zum Teil über die Grenzen einer Heidegger-Untersuchung hinwegsetzt. Neben der Eruierung der vom Philosophen aufgeworfenen Fragen werden zum einen die Konsequenzen in Betracht gezogen, die aus der These erwachsen, 
nach welcher sich zwischen dem Denken als diskursivem Kognitionsprozess und dem Dichten als ästhetischem Schaffensprozess ein spezifischer Übergang oder Grenzbereich postulieren lassen. Zum anderen setzt sich die vorliegende Studie zum Ziel, jener Kontroverse Rechnung zu tragen, die sich um Heidegger entfaltet hat, indem seine späten Schriften und Gedanken mit immer größer werdendem Unverständnis aufgenommen, und allmählich einer Grauzone der Wissenschaft zugerechnet wurden (Jahraus 2004, 18-22; Jaspers 2013, 253-386; Thomä 2013, XVI-XVII). Es wird jetzt dafür plädiert, dass Heideggers Denk- und Sprechweise in seinen späteren Texten nicht als der „Jargon der Eigentlichkeit“ abgetan werden dürfte, wie es Adorno in seinem Buch „Zur deutschen Ideologie“ (Adorno 1964) getan hat, sondern sie sollte gerade als die konsequente, der analytischen Erkenntnistheorie trotzende, aber nicht immer auf Anhieb problemlos nachvollziehbare Zuendeführung seiner sprach- und kunstphilosophischen Ideen betrachtet werden.

\section{Die Problematik und Bedeutung des Ursprungs}

Martin Heidegger hat zwar seine Thesen zur Beschaffenheit und Funktion der Dichtung in dem Aufsatzband „Unterwegs zur Sprache“ (Heidegger 2007) dargelegt, er widmete sich jedoch diesem Themenkreis auch in anderen Texten ${ }^{1}$, um das Wesen des Problems, trotz dessen oft verschwommener Konfusität beleuchten zu können. Wie es schon vorausgeschickt wurde, steht im Zentrum der vorliegenden Arbeit eine Untersuchung, die dieses Problem unter die Lupe nimmt, und danach fragt, inwieweit dem Dichten als ästhetischem Prozess diskursiv-kognitive Funktionen zugesprochen werden können?

Gehen wir vom Axiom der Heideggerschen Philosophie aus, nach welchem das Dichten als die früheste Realisierung des Mythos (Geisenhanslüke 2015, 9-12) die ursprünglichste Form des Nachdenkens über die Welt und das menschliche Leben darstellt, während die Reflektierung des Lebens als ,wissenschaftliches' Phänomen bereits mit dem Logos gleichzusetzen ist, und sie gehört in wissenschaftshistorischer Hinsicht einer späteren Entwicklung an. Die dichterische Sprechweise, wie sie bei Heidegger thematisch wird, ist immer vor dem Hintergrund dieses Mythos-Logos-Konfliktes (Kirk-Raven-Schofield 2001, 8-33) zu verstehen. Heideggers Motivation, die beiden Kräfte miteinander harmonisieren zu wollen, lässt sich als eine Art Lebensaufgabe des Philosophen auslegen, der er jedoch nie richtig nachkommen konnte, denn er fiel immer wieder auf eine klare Anti-Logos-Einstellung zurück. Der Zusammenhang von Denken und Dichten und die Überbetonung des Dichtens dabei (Heidegger 2007a; Heidegger 2007b; Heidegger 2007c), fungierten für Heidegger mehr als nur Grundsätze und zurückkehrende Motive seines Philosophierens, sie begründeten gleichsam die Basis und Architektonik seines ganzen Denkens, von denen, trotz ihrer Metamorphosen die späte Philosophie von ihm determinierend mitbegründet wurde.

1 „Holzwege“(1950); „Der Ursprung des Kunstwerks“ (1959); „Unterwegs zur Sprache“ (1959) „Erläuterungen zu Hölderlins Dichtung“ (1971); „Zur Erfahrung des Denkens“ (1983). 
Als eine Art Propädeutik zur Heideggers Dichtungs-Auffassung leitet uns die Frage nach der. sog. dichterischen Sprechweise über, welche Heidegger von eineigen Ausnahmen ${ }^{2}$ abgesehen immer wieder an konkreten Werken von Hölderlin, Rilke, Trakl, und George darzulegen pflegte, ohne jedoch dem Leser eine theoretisch fundierte Basis für diese Untersuchungen mit auf den Weg zu geben. Der Forscher fühlt sich mit den äußerst gewöhnungsbedürftigen Heideggerlektüren zu den großen deutschen Dichtern und Denkern nicht selten sehr allein gelassen, weshalb es bei diesem Philosophen besonders empfehlenswert sein kann, im Sinne Ludwig Wittgensteins eine Leiter - ein Werkzeug - zu beschaffen, die danach weggeworfen werden kann, dass man auf ihr hinaufgestiegen ist (Wittgenstein 2006, 85), und die gesuchte Antwort gefunden hat. Die Leiter, auf welcher wir zu einer, wenn auch vorläufiger Lösung vorzudringen gedenken, ist eine sprachliche, sprachgeschichtliche und vor allem Poetologische Untersuchung, die bei Heidegger als „die Frage nach der Ursprünglichkeit“ (Heidegger 1994a) thematisch wird.

Da den Ausgangspunkt des Heideggerschen Theorems die Behauptung der Ursprünglichkeit des Dichtens (Mythos) gegenüber dem logozentrischen Denken (Logos) bildet, sei an dieser Stelle dem Ursprünglichkeits-Begriff besondere Aufmerksamkeit geschenkt. (Heidegger 1994a, 60-66; Heidegger 2003, 225289). Um den Begriff wenigstens einigermaßen nicht zu missinterpretieren, muss zunächst das Bildhafte an ihm beobachtet werden, indem der ,Ursprung' im Sinne von Quelle oder Anfang temporal/geschichtlich und philosophisch interpretiert wird. Den Anfang zu dem man zurückkehren muss, wo das menschliche Wissen noch eine Einheit der verschiedensten Seins- und Wissenssphären bedeutete, bevor in ästhetisches Schaffen (Mythos) und kognitiv-diskursives Wissen (Logos) zerfiel, interpretiert Heidegger als das Zeitalter einer seelisch-geistigen Offenheit, die am Ende des 20. Jahrhunderts nur noch von den „Wenigen“ und von den „Seltenen" (Heidegger 2003, 11-20) wahrgenommen wird. Es dürfte daran selbstverständlich keine Zweifel bestehen, ob Heidegger sich selbst zu den Seltenen gerechnet hätte. Was inn allerdings gegenüber anderen Denkern auszeichnete, war sein Mut und seine Entschiedenheit den eingeübten Denkmustern den Rücken zuzukehren, und sich auf den Ursprung der Philosophie- und Kulturgeschichte besinnend die tradierten Antworten auf die Grundfragen des menschlichen Lebens ungültig zu erklären, oder mindestens in Frage zu stellen. Sein bis heute relevantes Programm war die Theorie der Destruktion der Philosophiegeschichte (Heidegger 2006, 19-27), die sich etwas später in Jacques Derridas verzerrter Umarbeitung als die Dekonstruktion eine riesige Karriere einheimsen konnte. Was die Vertreter der Gegenwart tun müssen, ist eben das, was Heidegger ihnen schon vorgemacht hat: sich auf den Ursprung (Heidegger 1996, 182-192) des Wissens rückbesinnen, wobei dieses Wissen weder als lebloses „,antiquarische(s)“ Wissen (Nietzsche 2002, 257-273), noch als psychologisierende „monumentalische“ Geschichtsbetrachtung (Nietzsche 2002, 308) missinterpretiert werden darf. Die Dichtung spielt bei der Rückkehr zum Ursprung gerade aus methodologischen Gründen eine eminente Rolle, denn sie hat als Vertreter des ursprünglicheren universellen

2 Als Ausnahmen sei an die Studien „Die Sprache”, und „Aus einem Gespräch von der Sprache” hingewiesen, in denen sich ein traditionell anmutendes Argumentationsschema entfaltet. 
Wissens die Funktion und die Möglichkeit inne, den im Zeitalter der Fachwissenschaften kaum mehr erkennbaren Zusammenhängen der menschlichen Existenz in ihrem Wesen wieder Transparenz zu verschaffen, und sie kognitiv fassbar zu machen.

\section{Ein dichtender Denker: Heidegger}

Nach Martin Heideggers Postulat zeichnet sich in der Sprache der Dichtung eine besondere Form des menschlichen Weltverhältnisses ab, denen er in vielen literarischen und poetologisch-ästhetischen Analysen nachgegangen war. Die Sprache der Dichtung mit ihrer potenzierten Metaphorizität und die sich diskursiv strukturierende (Alltags)Sprache werden in seinen Texten klar auseinander gehalten, und das sog. dichterische Sprechen als die Condition sine qua non des Problems aufgezeigt (Heidegger 2007b, 11-16). In dieser Form des Sprechens verbinden sich Denken und Dichten, Logos und Mythos in einer „Zwiesprache“ (Heidegger 2007c, 38), in der sich die gesuchte ursprüngliche Einheit vom dichtenden Denken und vom denkenden Dichten realisieren kann. Aber was versteht eigentlich Heidegger unter dieser von inm suggerierten Einheit, die auf dem ersten Blick etwas rätselhaft wirkt? Wie soll man Heideggers provokative Behauptungen verstehen, nach denen man zu denken verlernt habe, oder dass die Wissenschaft gar nicht denke (Heidegger 2004, 41-66)? Die Rezeption der späten Heidegger-Texte verläuft heute in zwei Richtungen: entweder schon die Art der Fragestellung wird als leere und fast illegitime Provokation eingestuft, und als trauriges Ergebnis des „mythopoetischen Irrgangs“ (Jahraus 2004, 22) des alten Philosophen diskqualifiziert, oder seine Texte werden von den Heidegger-Anhängern simplifizierend paraphrasiert, ohne dabei tieferes Erklärungspotenzial erzielen zu können.

Der hier gewählte Weg führt in diese Grauzone der Philosophie und der Literatur durch die Poetologie: die Texte eines denkenden Dichters und eines dichtenden Denkers werden jetzt nach jenen Zusammenhängen erforscht, die sich aus den Implikationen der Metaphorizität und der Begrifflichkeit im Bereich der Dichtung und der Philosophie ableiten lassen.

\section{Ein denkender Dichter: Rilke}

Im ersten Schritt wird am Beispiel eines anti-kartesischen Gedichts von Rainer Maria Rilke die Grundattitüde der untersuchten „dichterischen“ Sprechweise zu zeigen sein, wodurch der auto-reflexive Charakter des dichterischen Sprechens als die gesuchte Differentia specifica des Dichtens gegenüber dem Denken freigelegt werden kann.

Warum kann Rilkes frühes Gedicht „Ich fürchte mich so...“ (Rilke 2002, 188) als anti-kartesisch bezeichnet werden? René Descartes hat denjenigen Zustand als die Vollendung des menschlichen Denkens visioniert, in welchem der Mensch über eine vollkommene Sprache verfüge, die mit den Dingen der Welt vollkom- 
men kongruieren könne. (Descartes 2013, 45-52). Diese Kongruenz zwischen Logos und Kosmos bedeutet das Höchstmaß an Eindeutigkeit der Wörter, wobei es keine Definitionsmangel mehr besteht, denn alles in der reinen Begrifflichkeit aufgelöst und dadurch dem Erkennsubjekt transparent ist. Diese zum Ziel gesetzte Eindeutigkeit lässt keine alternativen Interpretationsmöglichkeiten gegenüber dem Logos zu, und eine Interpretation würde sich in der Epoche der völligen Eindeutigkeiten sowieso erübrigen. Die kartesische Vision einer kognitiv-reduktionistischer Utopie mag vielleicht auf dem ersten Blick als etwas Wünschenswertes erscheinen, aber in Rilkes Gedicht steigen dem lyrischen Subjekt im Zusammenhang mit einem solchen logozentrischen Zustand schon erhebliche Befürchtungen auf:

Ich fürchte mich so vor der Menschen Wort.

Sie sprechen alles so deutlich aus.

Und dieses heißt Hund und jenes heißt Haus,

und hier ist der Beginn und das Ende ist dort.

Mich bangt auch ihr Sinn, ihr Spiel mit dem Spott, sie wissen alles, was wird und war; kein Berg ist innen mehr wunderbar; ihr Garten und Gut grenzt grade an Gott.

Ich will immer warnen und wehren: Bleibt fern.

Die Dinge singen hör ich so gern.

Ihr rührt sie an: sie sind starr und stumm.

Ihr bringt mir alle die Dinge um.

\section{1. Die Hybris der Ratio}

Die durch die Partikel „so“ ausgedrückte Emphase im ersten Vers spricht von einer Furcht, deren Objekt das menschliche Wort ist. Die Emphase der Furcht gegenüber den menschlichen Machenschaften gehört bei Rilke - besonders in den späten Werkstufen - zu den Topoi seiner Dichtung. Die Furcht und die Angst beherrschen fast den ganzen Zyklus der „Duineser Elegien“, und in den „Aufzeichnungen“ - in seinem einzigen Roman - tritt die Angst als die eigentliche werkstrukturierende Komponente in poetischer Funktion auf. Das jetzt analysierte Gedicht bildet aber nach der Art der Thematisierung scheinbar eine Ausnahme. Das Objekt, das hier beim lyrischen Subjekt die Furcht auslöst, gehört nicht zur Sphäre der Irrationalität und der Transzendenz, es ist kein tief in der Psyche verankertes Phänomen. Das ist eben das „menschliche Wort“, mit seinen Implikationen, wie dem Sprechen und dem Denken. Das „Wort“ fungiert hier auf einer abstrakteren Ebene als ein Oberbegriff für die Differentia specifica des Menschenwesens, das als das einzige Lebewesen über die Fähigkeit des Sprechens verfügt. Deshalb dürfte es seltsam erscheinen, dass es ihm Furcht einflößen würde. Im zweiten Vers kommt es dann zur Erklärung der obigen Aussage.

„Sie sprechen alles so deutlich aus:“ 
Um diesen Satz richtig verstehen zu können, rufen wir uns ins Gedächtnis, was wir über die kartesische Forderung nach Eindeutigkeit des Urteilens und Sprechens bemerkt haben. Descartes hat in seinem Werk „Discours de la Méthode“vier Regeln aufgestellt, die das absolute Wissenserwerb garantieren sollen (Déscartes 2016, 32-34). Wir müssen uns jedoch nur der ersten Regel der kartesischen Methodenlehre hinwenden, welche die Klarheit und Bestimmtheit der Gegebenheiten betont, wodurch die Dinge des Lebens in klaren und deutlichen Begriffen aufgefangen werden können. Die erste Regel sagt aus: alles, was der Forderung nach Klarheit und der daraus resultierenden Begrifflichkeit nicht standhalte, müsse als undeutlich und undefinierbar verworfen werden. Rilke hat in seinen späteren Werken das oben skizzierte rationalistische Verhältnis zur Welt mit dem menschlichen Willen zur gedeuteten Welt umschrieben. Dieses Welt-Deuten steht nach der Rilkeschen Intention für die einseitige Vergegenständlichung und Simplifizierung der lebendig-bunten, generell uneindeutigen Welt, und versinnbildlicht dabei die „,veloziferische“3 Entfernung von den Ursprüngen der Kultur, die nach der Aussage des Gedichts in der Vergegenständlichung durch die Sprache kulminiert, und in letztem Vers mit einem metaphorischen Mord an den echten Dingen der Welt gleichgesetzt wird: „Ihr bringt mir alle die Dinge um.“

Die beiden letzten Verse der ersten Strophe unterstreichen in den fast rituellen Nennungen jenen vergegenständlichenden Deutlichkeitsanspruch, der das Verhältnis des Menschen zu seiner Welt charakterisiert, und das lyrische Subjekt langsam zur Verzweiflung bringt. Auch dem Prozess der Nennung der Dinge wird bei Rilke eine, die Grundbedeutung weit übersteigende Funktion zugesprochen. Durch die Nennung werden die Dinge der Welt wie Katalog-Gegenstände aufgezählt, und in Ordnung gesetzt. Es ist natürlich fraglich, was diesen postulierten Ding-Ordnungen Gültigkeit verleihen kann, und wie sie sich zu einer Episteme ordnen können? Mit dem Foucaultschen Vokabular ausgedrückt, geht es bei der Nennung der Dinge um anonyme Ordnungen und Strukturierungen des Wissens, oder um dessen Geltungsrahmen (Münker-Roesler 2000). Im Vers 3 werden zuerst ein Lebewesen (,Hund“) und ein Gegenstand (,Haus“) genannt, dann im Vers 4 die abstrakten Phänomene „Beginn“ und „Ende“. Durch die in der ersten Strophe evozierte Sprechweise wird die Episteme des Descartesschen Zeitalters reflektiert, in dem die Begrifflichkeitsforderung als absoluter Maßstab gesetzt wird, während die ursprünglichere, dichterische Sprechweise (die Dichtung) an ihrer einstigen existenziellen Relevanz einzubüßen scheint.

Die zweite Strophe des Gedichtes bietet eine präzisierte Darstellung des menschlichen Denkens, das durch eine hybrisartige Kritiklosigkeit im Sinne Kants gekennzeichnet ist. Die Kantsche Kritik an der reinen Vernunft lässt sich in unserem Kontext als Warnung auslegen, die den Menschen aufmerksam mache müsste, die a priori gegebenen Grenzen und Regeln der Erkenntnis zu beachten, und diese nicht zu überschreiten zu versuchen. Alles was man über die a priori Anschauungsformen hinaus noch erkennen möchte, ist notwendig zum Scheitern

3 Ich entnehme die Goethesche Wortschöpfung „,veloziferisch” Manfred Ostens Faust-Studie „Fluch vor allem der Geduld"(2012). Goethe versuchte den immer rasender werdenden technologischen und zivilisatorischen Fortschritt (das 'Veloziferische') dichterisch-philosophisch mit diesem Adjektiv zu fassen. 
verurteilt. Wenn der Mensch es trotzdem versucht, wird er sich nur in unauflösbare Antinomien verwickeln müssen.

\section{2. Die dichterische Kritik der Vernunft}

Der Anfangsvers der zweiten Strophe (,Mich bangt auch ihr Sinn, ihr Spiel mit dem Spott")

wiederholt die am Gedichtsanfang beschriebene Furcht in einer modifizierten und präzisierten Form. Jetzt wird auch „,der Sinn“ des menschlichen Wortes als gesondertes Furchtobjekt angegeben. Um den Gedanken hinter der Differenzierung zwischen „Wort" und „Sinn“ nachvollziehbar zu machen, soll die semantische Funktion von „Sinn“ in der Struktur des Gedichtes beleuchtet werden. Die althochdeutsche Grundbedeutung von „Sinn“ war ,Weg', ,Reise', ,Gang', und geht auf die indogermanische Wurzel ,sent-' ,eine Richtung nehmen' zurück. Wenn es dem lyrischen Subjekt vor dem „Sinn“ der menschlichen Worte bangt, dann wird eben jene Richtung befürchtet, in welche die schon analysierte veloziferische Denkweise den Menschen geführt hat. Diese Richtung des Denkens wird hyperbolisch „Spiel mit dem Spott" genannt, wobei der Spott mit dem Hohn gleichzusetzen ist, der für die Grundattitüde des überrationalisierten modernen Menschen charakteristisch ist. Das höhnische Spiel mit den Dingen der Welt zeugt von jener Bedenkenlosigkeit, die sich in der Anmaßung des Menschenwesens niederschlägt. Was der Mensch sich - seinem Erkenntnisvermögen - zutraut, wird in den folgenden Versen transparent gemacht.

sie wissen alles, was wird und war;

Kein Berg ist innen mehr wunderbar;

Ihr Garten und Gut grenzt grade an Gott.

Der befürchtete Totalitätsanspruch der Ratio schlägt sich im Behaupten des Allwissens nieder, das aber zu einem statischen, abgegrenzten, in tadellos definierten Begriffen aufgefangenen, aber leblosen Weltbild führt. Dieses Idealbild des absoluten Wissens steht im strikten Gegensatz zum Kantschen Theorem über die a priori Begrenztheit jeglicher Anschauungsformen der menschlichen Erkenntnis. Die Beschaffenheit des menschlichen Wahrnehmungshorizonts ist durch die a priori Anschauugsformen bedingt und dadurch unausweichlich begrenzt.

Die Neugierde und der Drang nach unbegrenztem Wissen sind aber feste Begleiter des Menschen. Ohne die Neugierde und die Fähigkeit des Staunens über die wunderbare Beschaffenheit der Welt sind die Entwicklung und die Erkenntnis unvorstellbar. Denken wir an die berühmte Textstelle im I. Buch der „Metaphysik“ des Aristoteles (Aristoteles 2001, 982b): „Weil sie sich nämlich wunderten, haben die Menschen [...] zu philosophieren begonnen." Das Staunen drängt den Menschen zum Erkennen-Wollen. Der Satz: „Kein Berg ist innen mehr wunderbar“ verweist auf eine epistemologische Situation des Menschenwesens, in der die Gleichgültigkeit und die Blindheit der Methoden herrscht, für deren Beschreibung Heidegger das Wort Gestell einführt (Heidegger 2002b, 37-47). Das Gestell versinnbildlicht die zum Stillstand gebrachte Bewegung der Welt, die sich von ihrem 
Ursprung weit entfernt hat. Über dieser Welt thront der Descartessche Geist, von dem nur das logisch richtige Sprechen akzeptiert wird, in welchem die Dinge klar und distinktiv formuliert in abgeschlossene Definitionen gedrängt werden. Diese existenzielle Situation ist es, was dem lyrischen Subjekt Angst einjagt.

\section{3. Der Gesang der Dinge}

Der deskriptiv geführte Duktus der ersten zwei Strophen wird in der dritten Strophe durch einen emotional gefärbten, präskriptiven Duktus abgelöst. Die dichterische Sprechweise dient jetzt nicht mehr zum ironischen Reflektieren der kartesisch-diskursiven Sprechweise, sondern dem Erzielen einer erwünschten Ursprungssituation, in der das Erstaunen über die Dinge der Welt noch gegeben war. Die gemeinsame bestimmende Komponente der beiden zentralen metaphorischen Wortgebilde ist das Wort „Ding“. Es wird in zwei Verbindungen verwendet: 1. die singenden Dinge; 2. das Töten dieser Dinge.

Die Dinge singen hör ich so gern.

Ihr rührt sie an: sie sind starr und stumm.

Ihr bringt mir alle die Dinge um.

Das Anrühren der Dinge zieht die Konsequenz nach sich, dass deren ursprünglich lebendiges Wesen zu leblosen (,starr“, „stumm“) Gegenständen verunstaltet (,umbringen“) wird. Besonders spannend erweist sich die Frage bei den Dingen, die man ab ovo ohne Gegenstandsbezug (der Gott, das Sein, der Tod, das Ding) zu begreifen und zu verstehen pflegt. Statt die Dinge begrifflich fassen zu wollen - sie anzurühren und umzubringen -, empfiehlt uns Rilke, sie singen zu lassen.

Damit Rilkes Metapher begreiflich wird, soll auf die metaphorische Struktur der menschlichen Erkenntnis in Bezug auf das „Wesen“ der Dinge hingewiesen werden. Wie schon mit Anlehnung an Kant dargelegt wurde, kann der Mensch über die „Dinge an sich“ keine Anschauung haben. Wenn wir die Dinge an sich nicht zu erkennen vermögen, besteht noch immer die Möglichkeit unser Verhältnis zu innen zu beobachten, und dieses Verhältnis zu thematisieren. Wenn die Dinge im Gedicht im Prozess des Singens erlebbar gemacht werden, müssen wir den dichterischen Prozess des Singens analysieren. Der Erkennende ist in diesem Fall der Hörer, der auf den Gesang der Dinge achtet, wodurch sie nicht verstummen müssen, sondern frei existieren können. Die akustische Metapher des Zuhörens bietet auch ex negativo eine weitere Deutungsmöglichkeit: dem Gesang der Dinge nicht zuhören, führt zu deren Verstummen. Das Verstummen der Dinge ist der Grund für ihre Unverständlichkeit, und für das daraus resultierende Unverständnis. Wenn man das Wesen der Dinge an sich (also die noumenalen Phänomene) in der Weise der kartesischen Begriffsbildung begreifen will, wird - aus den bereits dargestellten Gründen - scheitern müssen. Martin Heidegger hat die Fähigkeit, das Singen der Dinge hören zu können (=Dichtung=Kunst), in seinem poetischen Essay „Der Feldweg" als die eigentliche Voraussetzung des gesunden menschlichen Weltverständnisses aufgezeigt:

Aber der Zuspruch des Feldwegs spricht nur so lange, als Menschen sind, die, 
in seiner Luft geboren, ihn hören können. Sie sind hörige ihrer Herkunft, aber nicht Knechte von Machenschaften. [...] Die Gefahr droht, daß die Heutigen schwerhörig für seine Sprache bleiben. Innen fällt nur noch der Lärm der Apparate, die sie fast für die Stimme Gottes halten, ins Ohr. (Heidegger 2002a, 21)

\section{Das Geviert}

Nachdem Rainer Maria Rilkes Ideen zur zerbrechlichen Harmonie zwischen Kunst und Kognition auf der Grundlage seines Gedichtes analysiert worden sind, wird jetzt nach dem Wesen dieser Harmonie im Rahmen einer Analyse der metaphorisch überladenen Geviert-Lehre Martin Heideggers gefragt, die heute in der Forschung als das Geviert-Theorem in Evidenz gehalten wird. Das Geviert (Heidegger 2004, 157) lässt sich wohl nicht an einen konkreten Text binden, trotzdem empfiehlt sich den Aufsatz „Das Ding“ aus dem Jahr 1949 als jene Quelle zu betrachten, in der die zentralen Punkte der Theorems schon verhältnismäßig gebündelt vorkommen, wenn auch der Philosoph später in immer neuen Anläufen versucht hatte, seine Lehre weiter zu präzisieren und transparent nachvollziehbar zu machen.

Der Aufsatz „Das Ding“ gehört zu einem Gedankenkreis, dessen Texte um das Erfassen jener ursprünglichen Zusammenhänge ringen, die die menschliche Existenz in ihren Grundlagen bestimmen. Es handelt sich hier um die gesuchte ursprüngliche Einheit, in der sich die verschiedensten Seins- und Wissensgebiete nicht differenziert betrachtet werden, sondern im Zusammenhang eines reziproken Bezugssystems, das auf dem gegenseitigen Aufeinanderbeziehen vom Subjekt und Welt gegründet ist. Die Bedeutung eines Dinges lässt sich demnach ausschließlich aufgrund äußerlicher Dingbeschreibungen aus der Perspektive des Erkennsubjekts nie richtig bestimmen. Rilkes Gedicht vermittelte uns die gleiche Erkenntnis, wenn dort das lyrische Subjekt über die verstummenden und erstarrenden Worte berichtet, von denen die Dinge nur getötet werden. Mit dem Aufsatz „Das Ding“ hat Heidegger ein philosophisches Pendant zu Rilkes Gedicht entworfen, indem er das Geviert-Theorem ins Leben gerufen hat. Die Idee verfügt über eine besondere Erklärungskraft, denn in diesem Modell gelingt dem Philosophen die Einseitigkeit der vergegenständlichenden Weltdeutung dadurch zu überholen, dass sich in diesem System auch transzendente Komponente der Weltverhältnisstruktur (Gott, Glaube) berücksichtigen lassen, die sonst im diskursiv-logozentrischen Denken verloren gehen. Der Philosoph erklärt die Idee des Geviertes am Beispiel eines Dinges, - eines Kruges - wobei die Gegenseitigkeit zwischen den Polen des Bezugssystems metaphorisch und spielerisch als Nähe-Ferne-Parallelität zum Ausdruck gebracht wird. Trotz der ein wenig verstörend wirkenden Metaphorik lohnt es sich Heideggers resümierende Beschreibung des Kruges zu zitieren. Er fasst zunächst das Ziel seiner Methode zusammen: „Um das Wesen der Nähe zu finden, bedachten wir den Krug in der Nähe."(Heidegger 2004, 170). Die Formulierung ,das Ding in der Nähe zu betrachten' legt den Akzent auf die Nähe zwischen dem erkennenden Menschen und dem Objekt des Erkennens, wodurch das Verhältnis zwischen den beiden Polen ins Zentrum des Interesses gestellt wird. Das Wesent- 
liche des Erkennens besteht in der Qualität des Prozesses, in welchem sich der Mensch zum Ding in Bezug setzen muss, um es nicht bloß aus der Entfernung deskriptiv beobachten zu müssen. Er tritt in Kontakt zum Ding, um es zu innen verwandeln zu können, damit es im Sinne Rilkes singen kann. Heidegger setzt den Gedanken folgendermaßen fort:

Wir suchten das Wesen der Nähe und fanden das Wesen des Kruges als Ding. Aber in diesem Fund gewahren wir zugleich das Wesen der Nähe. Das Ding dingt. Dingend verweilt es Erde und Himmel, die Göttlichen und die Sterblichen; verweilend bringt das Ding die Vier in ihren Fernen einander nahe.[...] (Heidegger 2004, 170)

Zum Wesen des Dinges gelangt man nicht durch dessen einseitige Beobachtung, sondern durch die Beobachtung des Verhältnisses zu ihm, denn nur dann werden die Dinge nicht als intentionale Gegenstände der transitiven Weltdeutung behandelt, sondern als Dinge, deren Wesen in jener Nähe zu innen besteht, welche vom Subjekt des Weltverhältnisses selbst etabliert wird. Das ist die ästhetische Etablierung des Weltverhältnisses, wo das Erkennsubjekt und das Erkennobjekt in eine Nähe gebracht werden, in der die beiden aufeinander bezogen eine transzendentale Einheit bilden, um so verstanden zu werden. Dieses von Rilke in seinen Gedichten, von Goethe in seinen Dramen, oder von Thomas Mann in seinen Romanen realisierte Subjekt-Objekt-Verhältnis wird von Heidegger mit Hilfe der Geviert-Metapher erläutert. Er legt das Geviert symbolhaft als das Spiel der vier imaginativen Richtungen des menschlichen Denkens aus, wobei diese vier ou-topischen Richtungen vier Dimensionen darstellen, deren Wesen in ihrer Zusammengehörigkeit, in ihrer reziproken Struktur besteht, indem sie sich zeitlos, differenzlos und präsentisch aufeinander beziehen (Heidegger 2002, 15). Die vier Dimensionen des Geviert-Bezugsgeflechts sind 1) der Himmel 2) die Erde, 3) die Göttlichen und 4) die Sterblichen. Der Mensch, als Sterblicher ist ein Teil des Gevierts, und er muss für sich zwischen Himmel und Erde eine Stelle finden, um sich von seiner Daseinsangst befreien, und die ihm auferlegte Aufgaben bewältigen zu können. Um Heideggers Idee zur dynamischen Einheit der vier Dimensionen des Gevierts zu präzisieren, soll noch ein zweiter Auszug aus „Der Feldweg“ angeführt werden, wo die imaginativen Raumbezüge des Geviert-Theorems besonders gut zur Geltung kommen:

„Wachsen heißt: der Weite des Himmels sich öffnen und zugleich in das Dunkel der Erde wurzeln; dass alles Gediegene nur gedeiht, wenn der Mensch gleich recht beides ist: bereit dem Anspruch des höchsten Himmels und aufgehoben im Schutz der tragenden Erde." (Heidegger 2002a, 15) 


\section{Fazit Wozu Dichter?}

In der Heidegger-Forschung wird man den Eindruck nicht so einfach los, dass es dem späten Martin Heidegger eigentlich immer wieder nur um jene Frage geht, die er 1946 seinem Rilke-Aufsatz vorangestellt hat. Dort hat er mit Hölderlin gefragt: „Wozu Dichter in dürftiger Zeit?" Nun ja! Wozu Dichter in unserer Zeit? Was ist die Funktion der Dichtung? Hat sie überhaupt eine? Heidegger selbst hat darauf eine positive Antwort gegeben. Wie es in der vorliegenden Abhandlung ersichtlich wurde, misst der Philosoph der Dichtung eine Aufgabe zu, die von den sich diskursiv strukturierenden Wissenschaften, deren erkenntnistheoretische Basis in der Subjekt-Objekt-Differenzierung besteht, nicht bewältigt werden kann. Durch die Dichtung wird das sog. ästhetische Weltverhältnis konstituiert, das im Gegensatz zu dem auf den logozentrischen Differenzierungen basierenden diskursiven Weltverhältnis jene ursprüngliche Einheit anstrebt, die dem Menschen in der Welt eine Teilnehmerposition sichert, statt der Position des äußeren Beobachters in den diskursiven Wissenschaften.

Martin Heideggers dichtungstheoretische Ausführungen gehörten schon zur Zeit ihrer Abfassung zu den heiß diskutierten Theorien, und sie haben während der Jahre an ihrer Brisanz kaum etwas eingebüßt. Die lauten Protestbekundungen gaben langsam nach, die scharfe Ablehnung wurde auch zurückhaltender oder mindestens ein wenig differenzierter. Seine eigenwilligen Gedichtsanalysen stellen ihren Leser aber auch heute vor Herausforderungen. Einmal verblüffen sie inn mit ihrer Klarheit, ein andermal enttäuschen inn über ihre unwahrscheinlich dunklen Formulierungen. Der Leser darf aber dabei nie vergessen, worum es dem Philosophen geht. Er versucht seine Zuhörer auf die Dürftigkeit ihrer eigenen Zeit aufmerksam zu machen, indem über große Gedichte dichtend nachdenkt, und seine Leser zum freien Weiterdenken anregt.

\section{Literaturverzeichnis}

Adorno, Theodor Wiesengrund (1964): Jargon der Eigentlichkeit. Zur deutschen Ideologie. Frankfurt a. M.: Suhrkamp.

Aristoteles (2001): Metaphysik. Übers. Schwarz, Franz F., Stuttgart, Reclam.

Descartes, René (2016): Abhandlung über die Methode, richtig zu denken und Wahrheit in den Wissenschaften zu suchen. Übersetzt v. Kirchmann, Julis Heinrich von, Berlin: Holzinger.

Geisenhanslüke, Achim (2015): Die Wahrheit in der Literatur. Padeborn: Wilhelm Fink.

Heidegger, Martin ( $\left.{ }^{14} 2007 a\right)$ : Aus einem Gespräch von der Sprache. In Heidegger, Martin: Unterwegs zur Sprache. Stuttgart: Klett-Cotta, 83-155. p.

Heidegger, Martin ( ${ }^{3} 2003$ ): Beiträge zur Philosophie. Vom Ereignis. GA Bd. 65, Hrsg. v. Herrmann, Friedrich-Wilhelm von, Frankfurt a. M.: Klostermann. 
Heidegger, Martin ( ${ }^{32002 a): ~ D e r ~ F e l d w e g . ~ B e b i l d e r t e ~ S o n d e r a u s g a b e . ~ F r a n k f u r t ~ a . ~ M .: ~}$ Klostermann.

Heidegger, Martin ( $\left.{ }^{7} 1994 a\right)$ : Der Ursprung des Kunstwerks. In Heidegger, Martin: Holzwege. Hrsg. v. Hermann, Friedrich-Wilhelm von, Frankfurt a. M.: Klostermann, 1-74. p.

Heidegger, Martin ( $\left.{ }^{14} 2007 b\right)$ : Die Sprache. In Heidegger, Martin: Unterwegs zur Sprache. Stuttgart: Klett-Cotta, 9-34. p.

Heidegger, Martin ( $\left.{ }^{14} 2007 \mathrm{c}\right)$ : Die Sprache im Gedicht. In Heidegger, Martin: Unterwegs zur Sprache. Stuttgart: Klett-Cotta, 35-82. p.

Heidegger, Martin ( $\left.{ }^{10} 2002 b\right)$ : Die Technik und die Kehre. Stuttgart: Klett-Cotta.

Heidegger, Martin ( $\left.{ }^{19} 2006\right)$ : Sein und Zeit. Tübingen: Niemeyer.

Heidegger, Martin ( $\left.{ }^{7} 1994 b\right)$ : Wozu Dichter? In Heidegger, Martin: Holzwege. Hermann, Friedrich-Wilhelm von (Hrsg.). Frankfurt a. M.: Klostermann, 269-320. p.

Jahraus, Oliver (2004): Martin Heidegger. Eine Einführung. Stuttgart: Reclam.

Jaspers, Karl (2013): Notizen zu Martin Heidegger. Hrsg. v. Saner, Hans, München: Piper.

Kirk, Geoffrey S.-Raven, John E -Schofield, Malcolm (Hrsg.) (2001): Die vorsokratischen Philosophen: Einführung, Texte und Kommentare. Übersetzt v. Karlheinz Hülser, Stuttgart/Weimar: Metzler.

Nietzsche, Friedrich (2002): Vom Nutzen und Nachteil der Historie für das Leben. In Kritische Gesamtausgabe. Montinari, Carlo-Colli, Giorgio (Hrsg.). Abt. III. Bd. 1.

Osten, Manfred (2012): „Fluch vor allem der Geduld!“ - Zur Aktualität der Faust-Tragödie im Zeitalter der Globalisierung. In Bernáth, Árpád-Mitnyán, Lajos-Simon-Szabó, Ágnes (Hrsg.): Faust I und kein Ende. Studien zu Goethes Werk. Szeged: Grimm Verlag, 140152. p.

Rilke, Rainer Maria ( ${ }^{132002): ~ I c h ~ f u ̈ r c h t e ~ m i c h ~ s o . ~ I n ~ R i l k e, ~ R a i n e r ~ M a r i a: ~ D i e ~ G e d i c h t e . ~}$ Hrsg. v. Zinn, Ernst, Frankfurt a. M.: Insel, 188-189. p.

Thomä, Dieter (Hrsg.) (2013): Heidegger Handbuch. Leben-Werk-Wirkung. Stuttgart/ Weimar: Metzler.

Wittgenstein, Ludwig (2006): Tractatus logico-philosophicus. In Schulte, Joachim (Hrsg.) Werkausgabe, Bd. 1, Frankfurt a. M.: Suhrkamp, 7-85. p. 